\title{
Respiratory calcium fluctuations in low-frequency oscillating astrocytes in the pre-Bötzinger complex
}

Yoshitaka Oku ${ }^{1, *}$, Jens Fresemann ${ }^{2}$, Fumikazu Miwakeichi ${ }^{3,4}$, and Swen Hülsmann ${ }^{2,5, *}$

1 Department of Physiology, Hyogo College of Medicine, Nishinomiya, Hyogo, 663-8501 Japan

2 Clinic for Anesthesiology, Laboratory for experimental Neuroanesthesiology, University Hospital Göttingen, 37099 Göttingen, Germany

3 Department of Statistical Modeling, The Institute of Statistical Mathematics, Tokyo, 190-8562, Japan

4 Department of Statistical Science, School of Multidisciplinary Sciences, The Graduate University for Advanced Studies, Tokyo, 190-8562, Japan

5 DFG Research Center for Nanoscale Microscopy and Molecular Physiology of the Brain (CNMPB), Göttingen, Germany

* Corresponding authors:

Prof. Dr. Yoshitaka Oku: Department of Physiology, Hyogo College of Medicine, Nishinomiya 663-8501 Japan, Tel: +81-(0)798-45-6385; fax: +81-(0)798-48-9643.

E-mail address: yoku@ @yo-med.ac.jp

Prof. Dr. Swen Hülsmann: Clinic for Anesthesiology, Laboratory for experimental Neuroanesthesiology, University Hospital Göttingen, 37099 Göttingen, Germany Tel: +49-(0)551-39-9592; fax: +49-(0)551-39-9676.

E-mail address: shuelsm2@uni-goettingen.de 


\section{Abstract}

Astrocytes have been found to modulate neuronal activity through calcium-dependent signaling in various brain regions. However, whether astrocytes of the pre-Bötzinger complex (preBötC) exhibit respiratory rhythmic fluctuations is still controversial. Here we evaluated calcium-imaging experiments within preBötC in rhythmically active medullary slices from $\operatorname{TgN}($ hGFAP-EGFP) mice using advanced analyses. $13.8 \%$ of EGFP-negative cells, putative neurons, showed rhythmic fluorescent changes that were highly correlated to the respiratory rhythmic fluctuation (cross-correlation coefficient > 0.5 and $\mathrm{dF} / \mathrm{F}>0.2 \%)$. In contrast, a considerable number of astrocyte somata exhibited synchronized low-frequency $(<0.03 \mathrm{~Hz})$ calcium oscillations. After band-pass filtering, signals that irregularly preceded the calcium signal of EGFP-negative cells were observed in $10.2 \%$ of astrocytes, indicating a functional coupling between astrocytes and neurons in preBötC. A model simulation confirmed that such preinspiratory astrocytic signals can arise from coupled neuronal and astrocytic oscillators, supporting a concept that slow oscillatory changes of astrocytic functions modulate neighboring neuronal activity to add variability in respiratory rhythm. 
Keywords: neuron astrocyte interaction; calcium imaging; respiratory rhythm generation; preBötzinger complex; coupled oscillators 


\section{Introduction}

Astrocytes have been recognized to play active roles in various brain regions through bidirectional communication with neuronal circuits (Fellin, 2009), and are able to facilitate neuronal synchronization (Angulo et al., 2004; Tian et al., 2005). In the respiratory network, astrocytes have been shown to be vital for ongoing neuronal activity (Hulsmann et al., 2000) and they were postulated to play a crucial role in central chemoreception (Gourine et al., 2010). Astrocytes often show intrinsic calcium oscillations independent of neuronal activity that propagate to neighboring astrocytes and modulate neuronal activity by releasing gliotransmitters (Parri et al., 2001; Pasti et al., 1997; Scemes and Giaume, 2006) or regulating extracellular neurotransmitter concentration (Gomeza et al., 2003; Schnell et al., 2011). Slow intrinsic calcium oscillations have also been found in the pre-Bötzinger complex (preBötC) of mice using slice preparations (Hartel et al., 2009; Schnell et al., 2011). However, the roles these astroglial calcium oscillations play in the respiratory network have not been elucidated yet.

Recently, controversial data have been published for the preBötC network regarding the existence of calcium oscillations that are coupled to the neuronal activity. While the group of one of the authors of this paper (S.H.) reported respiratory rhythmic membrane 
currents in astrocytes of the neonatal mouse preBötC, without finding evidence for respiratory rhythmic calcium signals, Okada and Colleagues together with Y.O. shortly thereafter reported preinspiratory calcium signals in preBötC of rat (Okada et al., 2012; Schnell et al., 2011). In the present study, we therefore reevaluate calcium imaging experiments within the preBötC that have been conducted with a multifocal multi-photon microscope using Oregon green BAPTA-1 AM (OGB-1) as a calcium indicator, and transgenic TgN(hGFAP-EGFP) mice that express the green fluorescent protein in astrocytes, using rhythmically active medullary slices (Schnell et al., 2011). Although the data have been analyzed before (Schnell et al., 2011), different analysis methods were now used to allow automatized detection of time-lagged calcium signals that are correlated with the neuronal respiratory network activity. Interestingly, we could identify not only low frequency oscillation as described before (Hartel et al., 2009; Schnell et al., 2011), but also some irregularly occurring calcium fluctuations preceding neuronal activity in $10 \%$ of preBötC astrocyte. In order to gain insights in the potential physiological significances of such pre-inspiratory astrocytic calcium activity, we simulate the behavior of weakly coupled neuronal and astrocytic oscillators using a mathematical model. 


\section{Methods}

For this paper we used a dataset from hGFAP-EGFP transgenic mice that was collected for a previously published study (Schnell et al., 2011). Since analysis algorithms have been advanced since the publication has been submitted, and since there was a paper published showing opposing data from rats (Okada et al., 2012), we decided to reevaluate the mouse data. Methods different from an earlier publication (Schnell et al., 2011) are described in sections 2.3-2.6.

\subsection{Animal handling}

Animals TgN(hGFAP-EGFP) were bred in the animal facility of the University Hospital

Göttingen and treated in accordance with the guidelines of the German Physiological Society as well as the regulations of the State of Lower Saxony and the Federal Republic of Germany. The institutional permit number was T19.08. Neonatal mice $\left(\mathrm{TgN}(\mathrm{hGFAP}-\mathrm{EGFP})^{\text {GFEC-Fki }}\right.$; postnatal day 0-11) that have astrocytes in the respiratory network labeled with the enhanced green fluorescent protein (Grass et al., 2004; Nolte et al., 2001) were used. 


\subsection{Two-photon calcium imaging data collection}

Methods of respiratory-related rhythm generating medullary slice preparation and multi-cell bolus loading into the preBötC were as described previously (Schnell et al., 2011). Calcium imaging was performed using a two-photon laser-scanning system (TriMScope, LaVision BioTec, Bielefeld, Germany) with a 40x (0.8 NA) water-immersion objective lens (Zeiss; Oberkochen, Germany) (Winter et al., 2009). OGB-1 fluorescence was detected at $800 \mathrm{~nm}$ excitation wavelength through a YFP-filter (BP 511-551 nm), whereas EGFP-fluorescence was detected through a CFP-filter (475-500 nm) with $900 \mathrm{~nm}$ excitation wavelength (Winter et al., 2009). Images were taken using a CCD-camera (Ixon 885; Andor Technology, Belfast, Northern Ireland, or PCO; Sensicam QE; Kehlheim, Germany) at $0.1 \mathrm{sec} /$ frame, and exported to TIFF format for later analysis using Matlab (Mathworks Inc., Natick, MA, USA).

\subsection{Data processing and automatic cell detection}

Data were band-pass filtered (cutoff frequencies: $0.05-1 \mathrm{~Hz}$ ), and converted into $\mathrm{dF} / \mathrm{F} \%$ by dividing change of fluorescence intensity of each pixel $(\mathrm{dF})$ by the average fluorescence intensity of the initial 10 frames of each pixel (F). To generate overlay images that allow later analyses, i.e., localization and discrimination of putative neurons 
(EGFP-negative) and astrocytes that exhibit calcium fluctuations related to respiratory rhythm, we overlaid EGFP signal, OGB-1 fluorescence, and cross-correlation image. The cross-correlation image (CCI) was made using the average calcium signal of the entire frame as the reference function, then spatially-filtered ( $5 \times 5$ pixels). This spatially-filtered CCI was pseudo-color mapped in red to indicate cells with respiratory-correlated fluctuation. The spatially-filtered (5 x 5 pixels) EGFP signal was pseudo-color mapped in green to indicate GFAP-positive astrocytes. The raw OGB-1 fluorescence, averaged for the first 10 frames and spatially-filtered ( 5 x 5 pixels), was pseudo-color mapped in blue to indicate locations of both neurons and astrocytes that were labeled with OGB-1. Finally these three images were overlaid (see Fig. 3B).

The locations of astrocytes in a frame were automatically identified as follows. First, local maxima, whose EGFP fluorescence intensity was greater than the mean of the entire frame, were searched within the scope of $15 \times 15$ pixels. Then temporal changes of mean OGB-1 fluorescence of $7 \times$ x 7 pixels ROIs centered on the local EGFP-fluorescent maximum were calculated to obtain calcium transients of each astrocyte (EGFP-positive).

Both OGB-1 signals and absence of EGFP fluorescence were used to automatically identify locations of EGFP-negative cells (putative neurons). First, local maxima of 
OGB-1 fluorescence, whose intensity were greater than the mean of the entire frame, and EGFP fluorescence intensity was less than its mean of the entire frame were searched within the scope of $15 \times 15$ pixels. Then, temporal changes of mean OGB-1 fluorescence of $7 \times 7$ pixels ROIs centered on the local maximum were calculated to obtain calcium transients of each EGFP-negative cells.

\subsection{Detection of low frequency oscillations in EGFP positive cells}

To detect low frequency oscillations in EGFP positive cells, autocorrelation function was calculated with lags ranging between $-100 \mathrm{~s}$ and $100 \mathrm{~s}$ after low-pass filtering (cutoff frequency: $1 \mathrm{~Hz}$ ) for each EGFP positive cell whose fluctuation amplitude is greater than $0.4 \mathrm{dF} / \mathrm{F} \%$, which is about 10 times the baseline noise. Then the autocorrelation function was smoothed by applying moving time averaging (window width $5 \mathrm{~s}$ ) twice, and peaks were searched at the frequency range between $0.015 \mathrm{~Hz}$ and $0.05 \mathrm{~Hz}$. If multiple peaks were detected, then the highest peak was chosen for oscillation frequency of the cell.

\subsection{Estimation of out of focus light artifacts}

The original paper used a rolling ball algorithm from ImageJ to eliminate background 
fluorescence (Schnell et al., 2011). Here we compared the analysis with and without background subtraction, but additionally evaluated the influence of out of focus fluorescence artifacts originating from cellular compartments below or above the focal cell by determining the OGB-1 calcium signal in the soma as well as in the vicinity of the cell. Therefore, we set an 11x11 pixel ROI centered on the soma, and calculated the average calcium signal inside and four quadrants of outside the 7x7 pixels ROI (Fig. 1). The cellular calcium signal observed in the $7 \times 7$ pixels is the inherent cellular calcium signal $\left(\mathrm{F}_{1}\right)$ plus out of focus signal $\left(\mathrm{BF}_{0}\right)$, and the calcium signal observed outside the $7 \times 7$ pixels ROI is the signal emitted from the focal plane $\left(\mathrm{F}_{2}\right)$ plus $\mathrm{BF}_{0}$.

\subsection{Cycle triggered averaging of cellular calcium fluctuations}

Respiratory-rhythmic calcium fluctuation was obtained by averaging calcium transients of EGFP-negative cells whose cross-correlation with the entire frame is $>0.3$ (see top traces in Figs 2 and 3). Subsequently, cross correlation coefficients between the respiratory-rhythmic fluctuation and identified cells were estimated for the lag ranging between $-4 \mathrm{~s}$ and $4 \mathrm{~s}$. The maximum of cross correlation coefficients (CCmax) and the lag at which the cross correlation became maximal (LAGmax) were calculated for each cell. Statistical significance of CCmax was evaluated by assuming that cross correlation 
coefficients are asymptotically normally distributed. The p-value was computed by transforming the correlation to create a t-statistic having n-2 degrees of freedom, where $\mathrm{n}$ is the number of data. The null hypothesis that the cross correlation equals zero was rejected when the p-value was less than $\alpha / \mathrm{N}$, where $\alpha=0.05$ and $\mathrm{N}$ is the number of identified cells in one imaging area (Bonferroni correction). Cells with LAGmax $<-200$ ms were defined as preinspiratory, cells with LAGmax > $200 \mathrm{~ms}$ were defined as postinspiratory, otherwise cells were classified as inspiratory.

Peaks of the respiratory-rhythmic calcium fluctuation were detected where the signal $>$ mean $+2 \mathrm{SD}$ and the peak-to-peak distance $>2 \mathrm{~s}$. Subsequently cellular signals were cycle-triggered averaged around peaks $(0.8 \mathrm{x}$ peak-to-peak distance pre- and post-peaks). Fluorescent intensity data was expressed as $\mathrm{dF} / \mathrm{F} \%$ to allow semi-quantitative comparisons of signal amplitudes.

\subsection{Simulation of weakly coupled neuronal and astrocytic oscillators}

To test the prediction that weakly coupled neuronal and astrocytic oscillators can generate preinspiratory astrocytic signals, we simulated a rhythmic respiratory neuronal network that interacts with an astrocytic network having low-frequency oscillatory activity. As a simplest case, we first tested a case in which one pacemaker neuron 
interacts with a low-frequency oscillator by means of changes of the extracellular medium (transmitter release or altered neurotransmitter uptake). The mathematical model described by Butera and others (model 1 in (Butera et al., 1999a)) was used to model a pacemaker neuron. Bursting property of neurons in this model depends on the combination of the persistent sodium current conductance $G_{N a P}$ and the leak current conductance $G_{\text {leak }}$; here we set $G_{N a P}=2.5 \mathrm{nS}$ and $G_{\text {leak }}=2.8 \mathrm{nS}$, which results in rhythmic bursting behavior of the neuron.

Since we do not know the origin and the mechanism of slow astrocytic calcium fluctuation, we adopted a Rössler attractor (Rössler, 1976) as a model of low-frequency oscillator:

$$
\begin{aligned}
& \frac{d x}{d t}=(-y-z) \cdot s f \\
& \frac{d y}{d t}=(x+a y) \cdot s f \\
& \frac{d z}{d t}=(b+z(x-c)) \cdot s f
\end{aligned}
$$

where $a=0.1, b=0.4, c=4, s f$ (scaling factor) $=0.0002$. With these parameter values, the Rössler attractor behaves as a limit cycle oscillator with a period of $32 \mathrm{~s}$, which represents the low frequency oscillation observed in astrocytes (see Fig 6A, middle panel). The Rössler attractor (the astrocytic oscillator) is coupled with the pacemaker neuron via variables $x$ and $z$. We assumed that the variable $x$ represents the astrocytic 
calcium signal, which is modulated by an input from an inspiratory rhythmic neuron:

$$
\begin{aligned}
& \frac{d x}{d t}=\left(-y-z-I_{\text {ext }}\right) \cdot s f \\
& I_{\text {ext }}=g_{\text {ext }} \cdot s_{i}
\end{aligned}
$$

where the input gain $g_{\text {ext }}=20$, and $s_{i}$ is the gating variable driven by neuronal depolarization defined by Eq (3) in Butera et al. (Butera et al., 1999b). Further, we assumed that the variable $z$ causes an exogenous current on the neuron:

$$
I_{E X}=g_{E X} \cdot z \cdot\left(V_{m}-E_{E X}\right)
$$

where $V_{m}$ is the membrane potential of the neuron, the excitatory gliotransmitter conductance constant $g_{E X}$ is set to $0.05 \mathrm{nS}$, and the reversal potential of gliotransmitter-mediated currents $E_{E X}$ is set to $0 \mathrm{mV}$. Alternatively, we could assume reduction of an inhibitory current to induce a similar exogenous current.

The differential equations were solved numerically using the fourth-order Runge-Kutta equation with a step size of $0.05 \mathrm{~ms}$. However, the network state was saved at an interval of $20 \mathrm{~ms}$, which was sufficient for tracing the subthreshold membrane potential trajectory. All algorithms were implemented in double precision routine in C\#.NET language and run on a 64-bit Pentium-based Windows 7 professional computer. 


\section{Results}

1949 EGFP-negative cells (presumably neurons) and 873 EGFP-positive astrocytes that where loaded with OGB1-AM were identified from 92 different area stacks (10 $\mu \mathrm{m}$ step size) within the preBötC of 9 slices.

\subsection{Low frequency oscillation in astrocytes of the preBötC}

From the 873 astrocytes, $479(54.9 \%)$ showed low frequency oscillation $(0.023 \pm 0.010$

$\mathrm{Hz}$ ) with an amplitude $>0.4 \mathrm{dF} / \mathrm{F} \%$ (e.g. Fig. 2 cells \# 3, 4, 5). Importantly, these astrocytic oscillations were highly synchronized within preBötC (Fig. 2B). In contrast, low frequency oscillation was rarely observed in EGFP-negative neurons/cells. It is appealing to speculate that these slow and irregular calcium oscillation might reflect functional changes within the astrocytic network that have some effects on the neuronal activity of neighboring neurons, since the peak amplitude and the interval of the respiratory calcium rises in neighboring neurons were variable (Fig. 2). With bicuculline and strychnine, there was a slight but insignificant increase (from $48.6 \%$ to $58.6 \%$ ) in astrocytes with low frequency oscillations as detected by autocorrelation.

Interestingly, the low-frequency fluctuation was considerably less or not detectable in the vicinity of the cell (the light blue line in Fig. 2, cells \#3 and \#4), indicating that this 
fluctuation originated directly from the astrocytes. Band-pass filtering (cutoff frequencies: $0.05-1 \mathrm{~Hz}$ ) unveiled higher frequency oscillation of astrocytes, some of which appeared to be coupled with the respiratory rhythmic calcium fluctuation (Fig. 3 cells \# 1, 4, 5).

\subsection{Automatic data analysis}

To detect the correlation between cellular calcium fluctuations and the respiratory rhythmic calcium fluctuation, phase shifts were taken into consideration. We calculated and evaluated the cross correlation function with lags ranging between $-4 \mathrm{~s}$ and $4 \mathrm{~s}$ (Oku et al., 2007). We then evaluated p-values of CCmax, the cross correlation with the lag that gave the maximal cross correlation coefficient. When phase shifts were taken into account OGB-1 intensity fluctuations of 1867 / 1949 GFAP-negative (96\%) cells and 795 of 873 EGFP-positive cell $(91 \%)$ were significantly correlated with respiratory rhythm ( $\mathrm{p}<0.01$, t-test with Bonferroni's correction).

The cross-correlation histogram of EGFP-negative, putative neurons had a single peak at 0.7 (Fig. 4A), suggesting that they are a rather homogenous population of respiratory neurons, including however also neurons whose calcium transients were loosely but significantly coupled with the respiratory rhythm. In contrast, the 
cross-correlation histogram of EGFP-positive astrocytes had two peaks (Fig. 4B), with a maximum at 0.3 . This might reflect a distinct astrocytic population whose calcium activity was correlated with the respiratory rhythm (a small peak at 0.5 ).

To further classify respiratory-correlated cells, we created lag histograms of EGFP-positive cells and EGFP-negative cells for LAGmax, the lag where the cross correlation between cellular calcium transients and the respiratory rhythmic calcium fluctuation became maximal (Fig. 4C and D). The lag histogram of EGFP-negative cells had, as expected, a single narrow peak at lag $=0$, suggesting that most EGFP-negative cells had inspiratory-modulated bursts of action potentials leading to calcium elevation. On the other hand the lag histogram of EGFP-positive cells had a much broader distribution, and besides the peak at lag $=0$, it had a small peak at lag $=2 \mathrm{~s}$, suggesting a small but distinct pre-inspiratory astrocyte population. When we define cells with LAGmax <-200 ms as pre-inspiratory, cells with LAGmax > $200 \mathrm{~ms}$ as post-inspiratory, and cells with LAGmax between -200 ms and $200 \mathrm{~ms}$ as inspiratory, 81/795 (10.2\%) respiratory-correlated EGFP-positive astrocytes were pre-inspiratory, 92 (11.6\%) post-inspiratory, and $622(78.2 \%)$ as inspiratory. Regarding EGFP-negative cells, 58 / $1867(3.1 \%)$ respiratory-correlated neurons only were pre-inspiratory, 43 (2.3\%) were post-inspiratory, and $1766(94.6 \%)$ were inspiratory. However, automatized analysis 
picked up cells with small amplitudes (Fig. 4E), which might have resulted in overestimation.

\subsection{Description of cell types by cycle triggered averaging}

We performed cycle triggered averaging to further characterize the different cell types

(Fig. 5). The amplitude of the cycle-triggered averaging signal in EGFP-positive inspiratory cells was $0.18 \pm 0.40 \mathrm{dF} / \mathrm{F} \%$ as compared to the signal in EGFP-negative inspiratory cells $(0.29 \pm 0.60 \mathrm{dF} / \mathrm{F} \%$; n.s. $)$. The rise time $(20 \%-100 \%)$ of the calcium signal in EGFP-positive inspiratory cells was $1.88 \pm 1.41 \mathrm{~s}$ as compared to the signal in EGFP-negative inspiratory cells $(1.66 \pm 1.24 \mathrm{~s})$. The decay time $(100 \%-20 \%)$ of the calcium signal in EGFP-positive inspiratory cells was $2.21 \pm 1.37 \mathrm{~s}$ as compared to the signal in EGFP-negative inspiratory cells $(2.11 \pm 1.24 \mathrm{~s})$. These kinetics of calcium signals were not significantly different between EGFP-positive and EGFP-negative cells. Furthermore, background subtraction using ImageJ eliminated inspiratory calcium signals in 444/622 EGFP-positive cells (e.g. Fig. 5 cell \#1-\#3), suggesting background fluorescence contamination. 


\subsection{Preinspiratory signals}

In a subset of astrocytes, a pre-inspiratory calcium signal became evident from the cross-correlation analysis (Fig. 5 cell \#4 shows a typical EGFP-positive putative preinspiratory astrocyte). Interestingly, this preinspiratory calcium signal was not regularly observed at every respiratory cycle, nor had it a constant shape. Nevertheless it resulted in the cycle-triggered averaging signal that preceded the inspiratory burst by $1.7 \pm 1.1 \mathrm{~s}$, and persisted after ImageJ background subtraction. 81 of 795 astrocytes showed this characteristic. Moreover, such preinspiratory activity was not observed in the vicinity of the cell (the light blue line in Fig. 5, cell \#4), indicating that the preinspiratory activity was not a contamination of background fluorescence from neighboring neuropil spreading above or below the focal plane, but rather originated from the astrocyte itself.

\subsection{Signals in the EGFP-negative cell}

268 of 1949 EGFP-negative cells, presumably neurons exhibited inspiratory fluorescent changes with cross-correlation coefficient $(\mathrm{CC})>0.5$ and amplitude $>0.2 \mathrm{dF} / \mathrm{F} \%$, which was about 5 times the baseline noise level. Mostly, in 126 of 1949 cells with CC $>0.7$, the inspiratory calcium signal was observed at every respiratory rhythmic calcium 
event. However, in some cells with lower CC values, an inspiratory calcium signal was only observed intermittently (Fig. 3, cell \# 8).

\subsection{Preinspiratory astrocytic activity can arise from weakly coupled oscillators}

Significant cross-correlation, with phase-advanced astrocytic calcium activity, does not necessarily imply the causality between neuronal and astrocytic activities. Rather, it is unlikely that astrocytic calcium signals with low-frequency fluctuation trigger inspiratory neuronal activity. Therefore, we hypothesized that such preinspiratory astrocytic calcium signals arise from weakly coupled neuronal and astrocytic network activity. To explore this possibility, we simulated the behavior of a coupled oscillator model consisting of a rhythmically bursting Butera-model neuron and a slowly oscillating Rössler-attractor astrocyte. As shown in Fig. 6, the rhythm of the bursting neuron is modulated by the astrocytic oscillatory activity, and the slow astrocytic oscillation is partially suppressed by neuronal bursts, which is clearly visible after filtering the low-frequency oscillation. In this situation, the astrocytic oscillator is weakly reset at every neuronal burst (type 1 resetting (Glass and Mackey, 1988)). As a consequence, the cycle-triggered averaged astrocytic activity precedes the neuronal activity, and classified as 'preinspiratory'. 


\section{Discussion}

We found that a subset (10.2\%) of GFAP-positive putative astrocytes exhibited rhythmic calcium elevations preceding inspiratory neuronal activity with a time lag that peaked at approximately $2 \mathrm{sec}$, which is consistent with the report of Okada et al. (Okada et al., 2012). The model simulation showed that such preinspiratory astrocytic activity could arise from weakly coupled neuronal and astrocytic oscillators.

\subsection{Technical considerations}

Schnell et al. (Schnell et al., 2011) reported that astrocytic rhythmic calcium signals were not detectable even though current transitions in a small number of preBötC astrocytes were found. Moreover, whole cell recordings combined with calcium imaging did reveal calcium fluctuations that coincided with rhythmic neuronal discharges. Furthermore, analysis in that paper involved the Rolling Ball Background Subtraction (ImageJ "rolling ball" algorithm; radius 30-50 pixel), which not only allowed to correct for illumination inhomogeneity (Bootman et al., 2013; Sternberg, 1983) but might also reduce out of focus light. On the other hand, rhythmic calcium oscillation might have been interpreted as irrelevant in the Schnell paper because of the fact that low-frequency $(<0.03 \mathrm{~Hz})$ fluctuations characteristic of astrocytes and high 
frequency noise were not removed by filtering (Schnell et al., 2011) and thus the signals were masked by noise or simply too small to be detected, since noise reduction was performed only by cycle triggered averaging. The paper further mentioned that in some astrocytes (see cell \#5 in Fig. 2,3 and 5) "rhythmic $\mathrm{Ca}^{2+}$-signals overlapped astrocyte (EGFP) fluorescence. However, the signals might have originated from neuronal processes located in the vicinity of the astrocytes, because cross-correlation activity maps (CC; see methods) only partially overlapped with EGFP-fluorescence. Of greater significance was that the temporal signature of the calcium transients was indistinguishable from a neuronal calcium signals".

In our reanalysis of the dataset, we found astrocytic rhythmic calcium fluctuations by statistical measures after high-pass filtering, which removed the typical and relevant low-frequency $(<0.03 \mathrm{~Hz})$ fluctuations characteristic of astrocytes and reduced high frequency noises (low-pass filter $1 \mathrm{~Hz}$ ). In the initial automatized Matlab algorithms, amplitude $(\mathrm{dF} / \mathrm{F})$ of the calcium signal was not analyzed as such, thus in a high number of cells, low amplitude $(\mathrm{dF} / \mathrm{F}<0.2 \%)$ fluorescence fluctuations were identified. Therefore, we assume that most of these signals are due to out of focus light contamination.

However, the analysis revealed also preinspiratory calcium rises in $10.2 \%$ of 
EGFP-positive putative astrocytes. Since this fluorescence fluctuation was either not observed or remarkably diminished in the vicinity of the cell, we assume that such preinspiratory calcium activity was not a contamination by out of focus activity. We are confident that this data is in line with the observation of putative preinspiratory astrocytes described by Okada et al. (8). As compared to signals in type 1 astrocytes in the Okada paper ((Okada et al., 2012), preinspiratory astrocytes in the present study lack inspiratory calcium rise (see Fig.5, cell \#4), whereas type 1 astrocytes have both preinspiratory and inspiratory calcium rises, supporting the notion that inspiratory calcium activity originated from neighboring neuropil (see below) .

Regarding the inspiratory calcium activity observed in astrocytes, firstly it should be noted that the null hypothesis is hardly rejected even if the cross-correlation coefficient is low $(<0.2)$, due to a large number of data points, up to 1600 taken per measurement. Secondly, we have to assume a high degree of contaminations, although the amplitude of calcium signals of ROIs centered on cells were greater than those in quadrants of the vicinity. For example, if axons or dendrites with strong respiratory rhythmic signals passed through the vicinity of the ROI, averaging neighboring calcium signals in all directions would dilute the signal intensity. Further, kinetics of inspiratory calcium signals observed in EGFP-positive cells is very similar to that seen in EGFP-negative 
cells. Finally, background subtraction eliminated inspiratory signal components in $71 \%$ of EGFP-positive, and $13 \%$ of EGFP-negative cells, respectively. Taken together, and considering the spatial resolution of a CCD camera-based detection system (Nipkow and TrimScope), a high number of false positive detections would have been included due to out-of-focus light. Further experiments, e.g. using genetically encoded calcium sensors, are necessary to overcome this problem.

\subsection{Functional relevance of respiratory-correlated astrocytic calcium fluctuations}

At present we cannot conclude that there is a causal link between the astroglial calcium signal and the neuronal activity. Indeed, cross-correlation does not imply any causality. For example, assuming a variable $a$ causes changes in both variables $b$ and $c$, even if there is no additional causality between $b$ and $c$, they will be correlated. Likewise, phase-advanced astrocytic calcium activity does not necessarily imply that astrocytes trigger the neuronal activity. Rather, it is unlikely that a slow oscillator (astrocytic network) drives a fast oscillator (respiratory neuronal network). Concordantly, our simulation shows that phase-advanced calcium activity could arise from coupled neuronal and astrocytic oscillators. In such situation, both neuronal and astrocytic networks generate intrinsic oscillations independently, i.e., astrocytic oscillation does 
not depend on neuronal drives, and neuronal oscillation does not depend on astrocytic drives; their coupling mutually modulate their intrinsic oscillations. Astrocytes are locally clustered and exhibit correlated calcium activity that can influence the neighboring neuronal activity in mouse hippocampus and neocortex (Sasaki et al., 2011). In the respiratory network, slowly oscillating astrocytes may also form a cluster and modulate the neighboring neuronal oscillator to add variability in respiratory rhythm. Such modulation might contribute to adaptation of respiratory network to changes in internal and external environments.

\subsection{Conclusions}

The authors agree on the existence of irregularly occurring preinspiratory calcium signals in astrocytes (like in type 1 cells Okada (Okada et al., 2012) without the inspiratory signal contamination) and the assumption that inspiratory calcium signals with neuron-like kinetics are most likely artifacts from neuropil. Taken together the data is in line with a novel concept that the intrinsic low frequency oscillations in preBötC astrocytes are coupled to the neuronal oscillator and thereby contributes to variability of the respiratory rhythm. 


\section{Acknowledgements}

This study was supported by JST strategic Japanese-German cooperative program in computational neuroscience, Grant-in-Aid for Scientific Research from the Japan Society for Promotion of Science (24300108 and 24500365) and the DFG (CNMPB, HU797/5-1 and HU797/8-1). The authors wish to thank Rishi Raj Dhingra (Salt Lake City) and Mathias Dutschmann (Melbourne) for fruitful discussion regarding the controversial astroglial calcium signals. 


\section{References}

Angulo, M.C., Kozlov, A.S., Charpak, S., Audinat, E., 2004. Glutamate released from glial cells synchronizes neuronal activity in the hippocampus. J Neurosci 24, 6920-6927.

Bootman, M.D., Rietdorf, K., Collins, T., Walker, S., Sanderson, M., 2013. $\mathrm{Ca}^{2+}$-sensitive fluorescent dyes and intracellular Ca2+ imaging. Cold Spring Harbor protocols 2013, 83-99.

Butera, R.J., Jr., Rinzel, J., Smith, J.C., 1999a. Models of respiratory rhythm generation in the pre-Botzinger complex. I. Bursting pacemaker neurons. J Neurophysiol 82, $382-397$.

Butera, R.J., Jr., Rinzel, J., Smith, J.C., 1999b. Models of respiratory rhythm generation in the pre-Botzinger complex. II. Populations Of coupled pacemaker neurons. J Neurophysiol 82, 398-415.

Fellin, T., 2009. Communication between neurons and astrocytes: relevance to the modulation of synaptic and network activity. Journal of neurochemistry 108, 533-544.

Glass, L., Mackey, M.C., 1988. From Clocks to Chaos. Princeton University Press, Princeton, New Jersey. 
Gomeza, J., Hulsmann, S., Ohno, K., Eulenburg, V., Szoke, K., Richter, D., Betz, H., 2003. Inactivation of the glycine transporter 1 gene discloses vital role of glial glycine uptake in glycinergic inhibition. Neuron 40, 785-796.

Gourine, A.V., Kasymov, V., Marina, N., Tang, F., Figueiredo, M.F., Lane, S., Teschemacher, A.G., Spyer, K.M., Deisseroth, K., Kasparov, S., 2010. Astrocytes control breathing through pH-dependent release of ATP. Science 329, 571-575.

Grass, D., Pawlowski, P.G., Hirrlinger, J., Papadopoulos, N., Richter, D.W., Kirchhoff, F., Hulsmann, S., 2004. Diversity of functional astroglial properties in the respiratory network. J Neurosci 24, 1358-1365.

Hartel, K., Schnell, C., Hulsmann, S., 2009. Astrocytic calcium signals induced by neuromodulators via functional metabotropic receptors in the ventral respiratory group of neonatal mice. Glia 57, 815-827.

Hulsmann, S., Oku, Y., Zhang, W., Richter, D.W., 2000. Metabolic coupling between glia and neurons is necessary for maintaining respiratory activity in transverse medullary slices of neonatal mouse. Eur J Neurosci 12, 856-862.

Nolte, C., Matyash, M., Pivneva, T., Schipke, C.G., Ohlemeyer, C., Hanisch, U.K., Kirchhoff, F., Kettenmann, H., 2001. GFAP promoter-controlled EGFP-expressing 
transgenic mice: a tool to visualize astrocytes and astrogliosis in living brain tissue. Glia $33,72-86$.

Okada, Y., Sasaki, T., Oku, Y., Takahashi, N., Seki, M., Ujita, S., Tanaka, K.F., Matsuki, N., Ikegaya, Y., 2012. Preinspiratory calcium rise in putative pre-Botzinger complex astrocytes. J Physiol 590, 4933-4944.

Oku, Y., Masumiya, H., Okada, Y., 2007. Postnatal developmental changes in activation profiles of the respiratory neuronal network in the rat ventral medulla. J Physiol 585, 175-186.

Parri, H.R., Gould, T.M., Crunelli, V., 2001. Spontaneous astrocytic $\mathrm{Ca}^{2+}$ oscillations in situ drive NMDAR-mediated neuronal excitation. Nat Neurosci 4, 803-812.

Pasti, L., Volterra, A., Pozzan, T., Carmignoto, G., 1997. Intracellular calcium oscillations in astrocytes: a highly plastic, bidirectional form of communication between neurons and astrocytes in situ. J Neurosci 17, 7817-7830.

Rössler, O.E., 1976. An Equation for Continuous Chaos. Physics Letters 57A, 397-398.

Sasaki, T., Kuga, N., Namiki, S., Matsuki, N., Ikegaya, Y., 2011. Locally synchronized astrocytes. Cerebral cortex 21, 1889-1900. 
Scemes, E., Giaume, C., 2006. Astrocyte calcium waves: what they are and what they do. Glia 54, 716-725.

Schnell, C., Fresemann, J., Hulsmann, S., 2011. Determinants of functional coupling between astrocytes and respiratory neurons in the pre-Botzinger complex. PloS one 6, e26309.

Sternberg, S.R., 1983. Biomedical Image Processing. IEEE Computer 16, 22-34.

Tian, G.F., Azmi, H., Takano, T., Xu, Q., Peng, W., Lin, J., Oberheim, N., Lou, N., Wang, X., Zielke, H.R., Kang, J., Nedergaard, M., 2005. An astrocytic basis of epilepsy. Nature medicine 11, 973-981.

Winter, S.M., Fresemann, J., Schnell, C., Oku, Y., Hirrlinger, J., Hulsmann, S., 2009. Glycinergic interneurons are functionally integrated into the inspiratory network of mouse medullary slices. Pflugers Arch 458, 459-469. 


\section{Figure Legends}

Figure 1. Measurement of background fluorescence. $F_{1}$ and $F_{2}$ represent intensities of inherent calcium fluorescence of 7 x 7 pixels ROI and its adjacent pixels, respectively. $\mathrm{BF}$ denotes background fluorescence. If $\mathrm{F}_{1}$ and $\mathrm{F}_{2}$ were time-invariant and did not show respiratory-related calcium fluctuations, $\mathrm{dF} / \mathrm{F}$ of the cell and its vicinity are $\mathrm{BF} /$ $\left(\mathrm{F}_{1}+\mathrm{BF}\right)$ and $\mathrm{BF} /\left(\mathrm{F}_{2}+\mathrm{BF}\right)$, respectively. See text.

Figure 2. Representative unfiltered calcium transients of EGFP-positive and negative cells. A: astrocytes are identified with GFAP promoter-controlled expression of EGFP, and B: cross-correlation image between each pixel and the low-frequency calcium oscillation of cell \#4 indicates that low-frequency astrocytic calcium oscillations are highly synchronized. Bar is $40 \mu \mathrm{m}$ length. C: top trace shows the respiratory rhythmic calcium fluctuation, and other traces show band pass filtered calcium signals of representative cells. For cells \#1 - 8, the location of each cell is indicated in left panels (A and B). For EGFP-positive cells (\#1-5), raw calcium signals of the cell (green) and its vicinity (light blue) are shown. 
Figure 3. Band pass filtered calcium transients of EGFP-positive and negative cells (without background subtraction) shown in Fig. 2. A: both neurons and astrocytes are stained with Oregon Green BAPTA-1 AM (OGB-1). B: overlay image in which EGFP, OGB-1, and cross-correlation coefficient signals are pseudo-color mapped in green, blue, and red, respectively. Bars are $40 \mu \mathrm{m}$ length. C: top trace shows the respiratory rhythmic calcium fluctuation, and other traces show band pass filtered calcium signals of identified cells. Through cells \#1 - 8, the location of each cell is indicated in the overlay image. For EGFP-positive cells (\#1-5), filtered calcium signals of the cell (green) and its vicinity (light blue) are shown. Red vertical lines indicate onsets of inspiratory neuronal activity. Note that the calcium transient of cell \#4 precedes the onset of inspiratory neuronal activity.

Figure 4. Cross-correlation histograms of EGFP-negative (A) and positive cells (B) are shown together with lag histograms of EGFP-negative $(\mathbf{C})$ and positive cells $(\mathbf{D})$. E: the relationship between cross-correlations and amplitudes of cycle-triggered averaged calcium fluctuations are shown where EGFP-negative cells are plotted in red and EGFP-positive cells are plotted in blue. 
Figure 5. Activation timings of cells represented in Figs 2 and 3 are evaluated by lagged cross-correlation images and cycle triggered averaging. Cross-correlations between filtered calcium transients of each pixel and the average calcium signal of the entire frame are pseudo-color mapped with lag $=0 \mathrm{~s}(\mathbf{A})$ and lag $=2 \mathrm{~s}(\mathbf{B})$. Bars are $40 \mu \mathrm{m}$ length. Note that the cross-correlation image with lag $=2 \mathrm{~s}$ revealed preinspiratory cells (e.g. cell \#4). Cycle triggered averaging data from cells are shown in $\mathbf{C}$ (without background subtraction) and D (with background subtraction). Each top panel shows the cycle triggered averaging signal of the respiratory rhythmic calcium fluctuation, and traces 1-5 are cycle triggered averaging signals of EGFP positive cell (green) and its vicinity (light blue). Note that inspiratory calcium signals in cells \#1-3 diminished after background subtraction, but the preinspiratory signal in cell \#4 remained.

Figure 6. Behavior of a coupled oscillator model consisting of a rhythmically bursting neuron and a slowly oscillating astrocyte. A: the top panel represents the membrane potential (magenta) and its moving-averaged sub-threshold potential (blue) trajectories of the bursting neuron, the middle panel represents trajectories of $x$ (green) and $z$ (light green, magnified x10) variables of Rössler-attractor astrocyte model, and the bottom panel represents the filtered trajectory of variable $x$. Note that respiratory rhythmic 
fluctuations are clearly visible after filtering the low-frequency oscillation. B: The top panel shows the cycle triggered averaging of the neuronal sub-threshold membrane potential, and the bottom shows cycle triggered averaging of the filtered signal of the astrocyte model (variable $x$ ). 
Figure 2

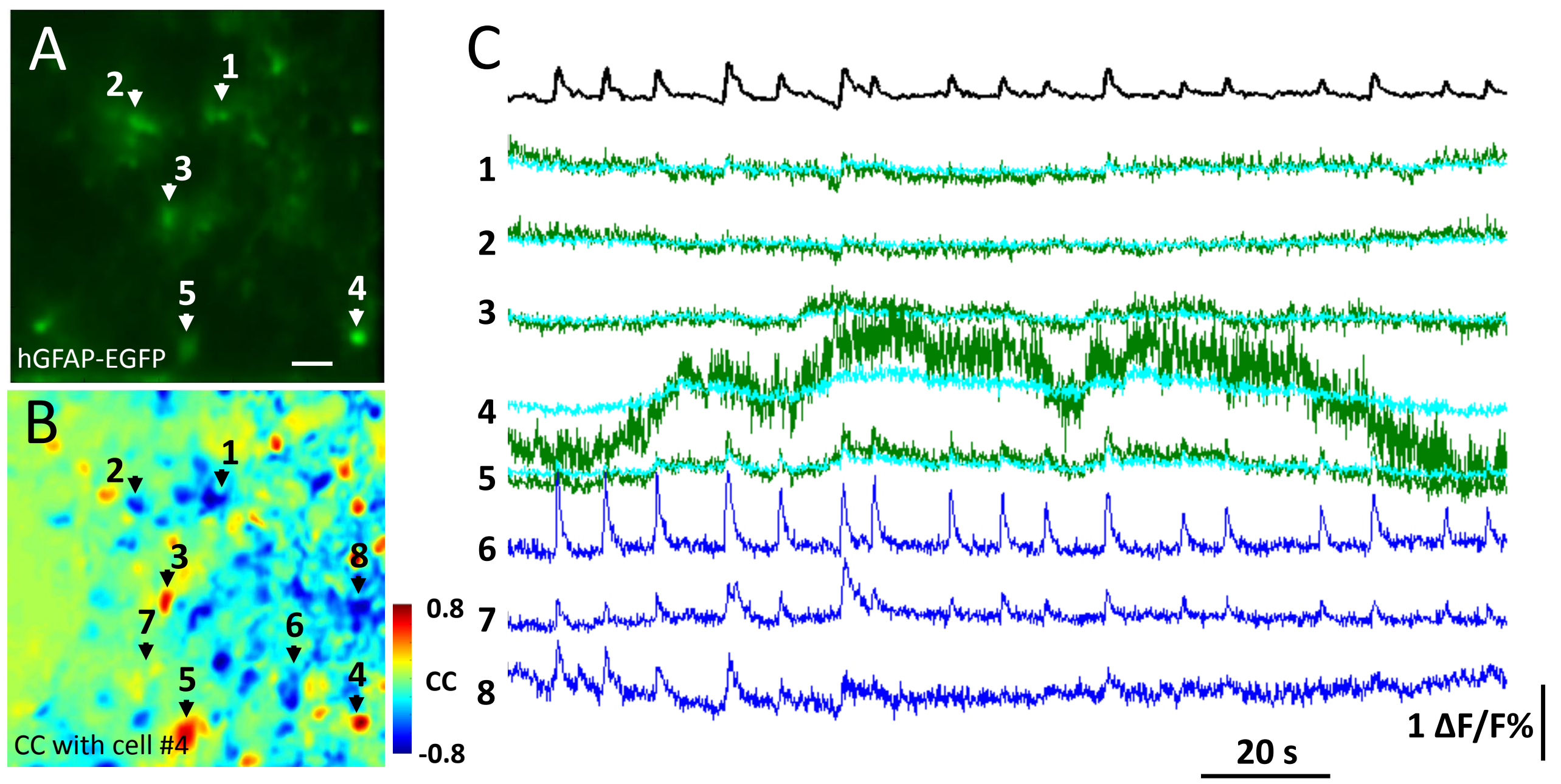


Figure 3



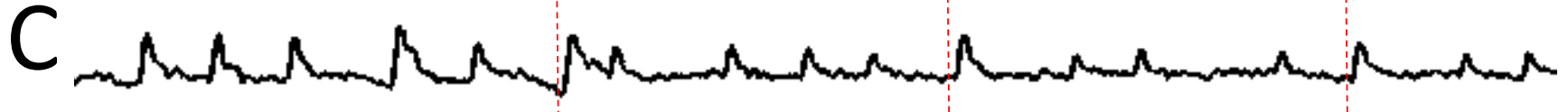

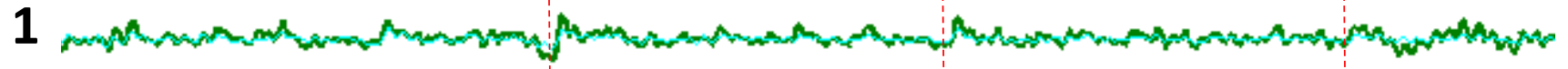

2

3

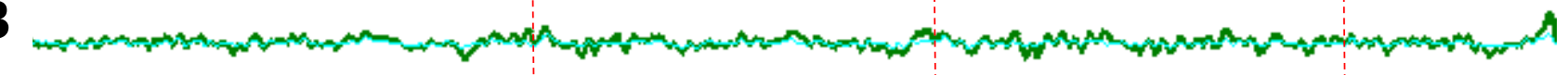

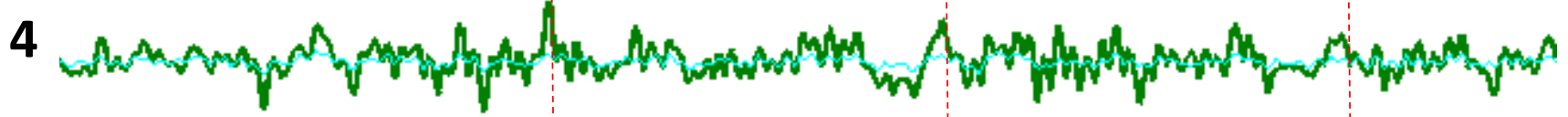



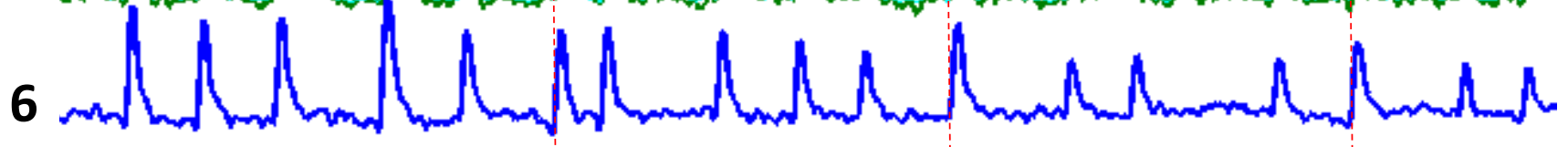

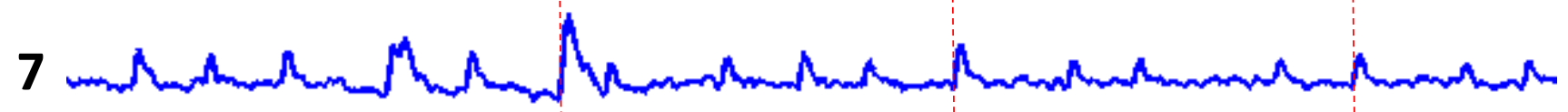

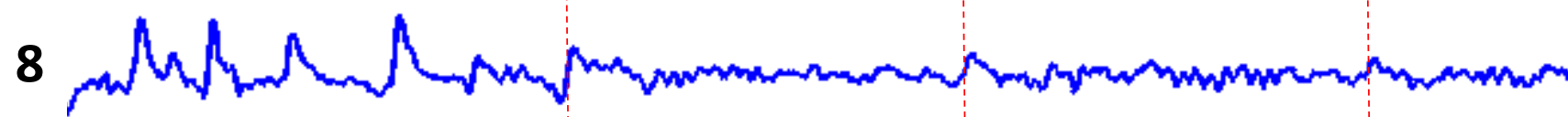
$20 \mathrm{~s} \quad 1 \Delta \mathrm{F} / \mathrm{F} \%$ 
Figure 4

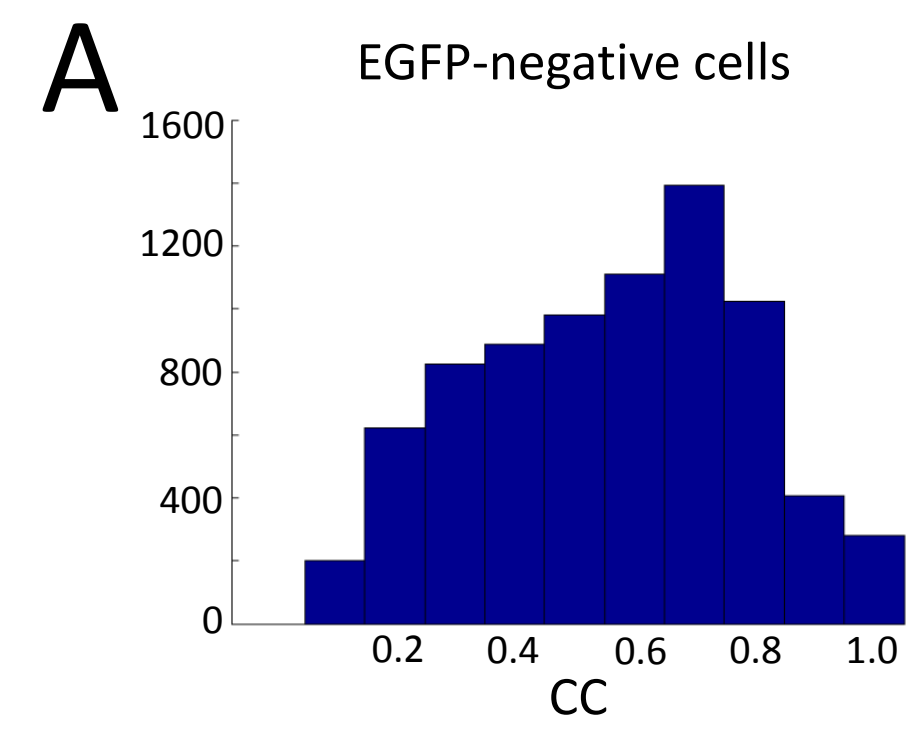

B



E

C

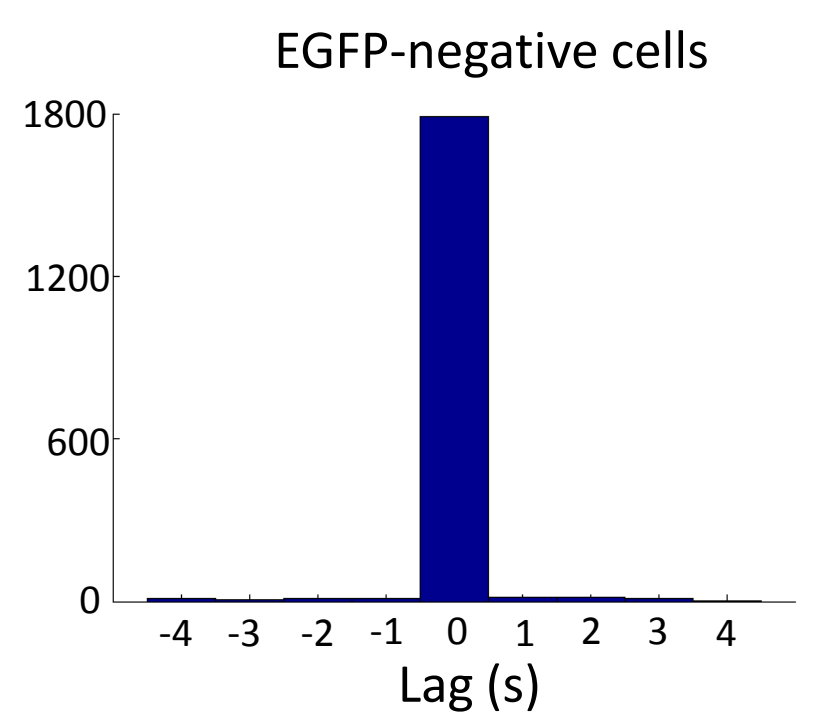

D

EGFP-positive cells




Figure 5

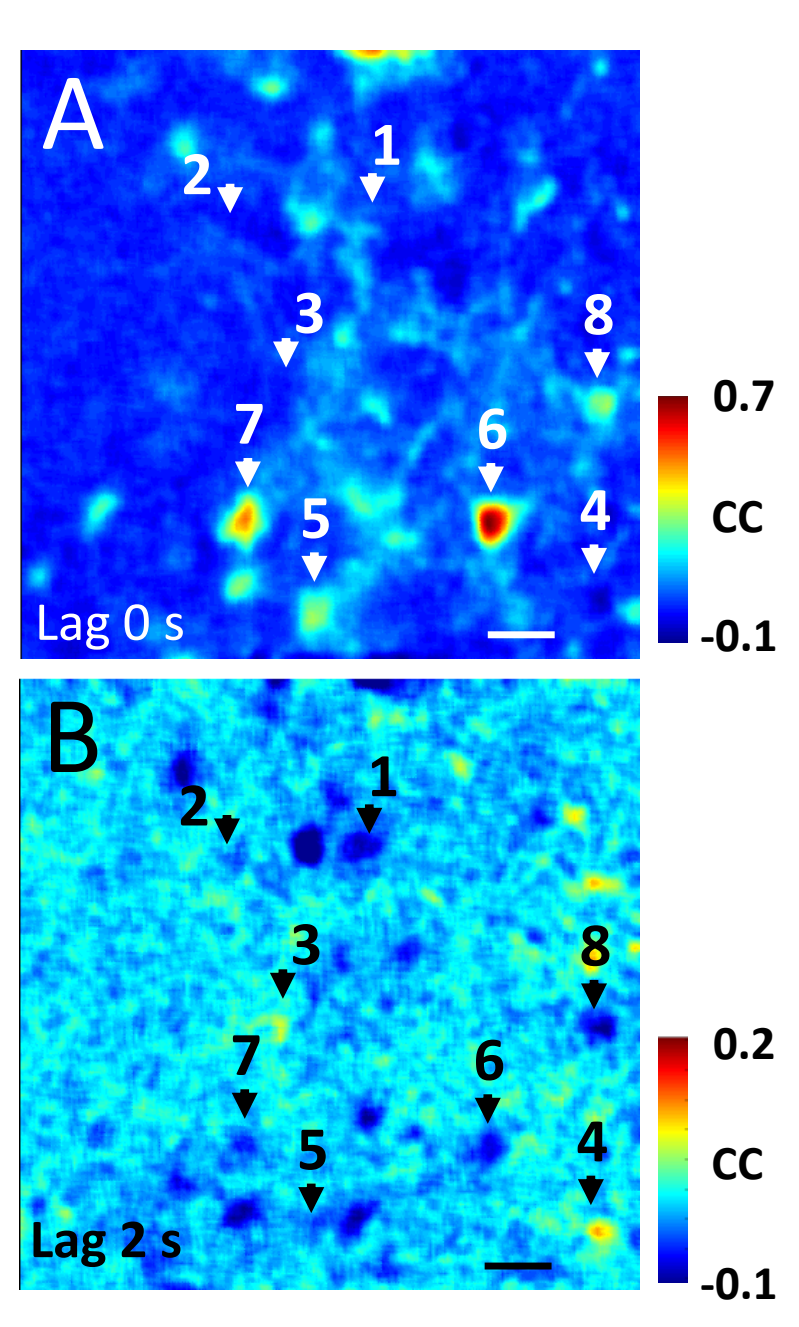

C Background Subtraction (-)

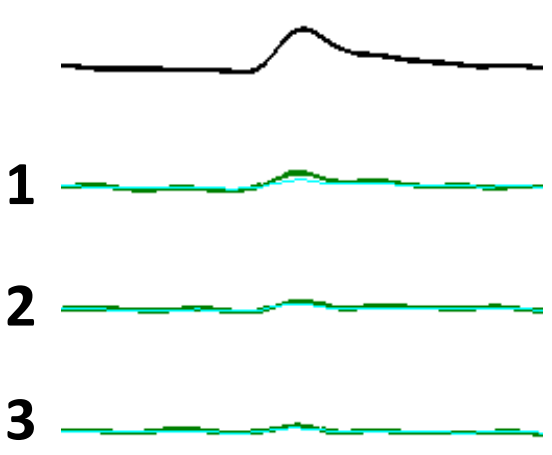

4

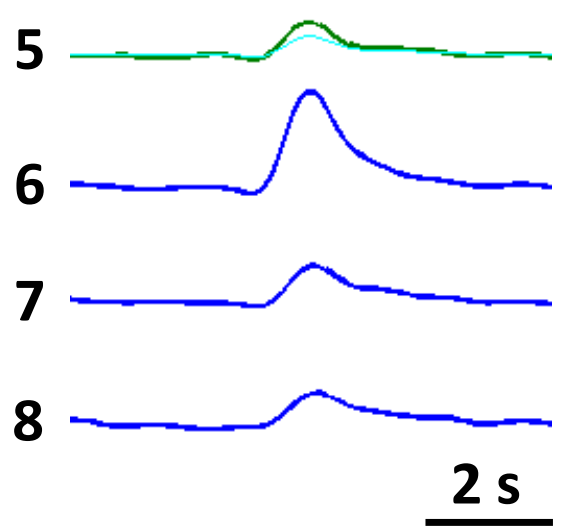

D Background Subtraction (+)
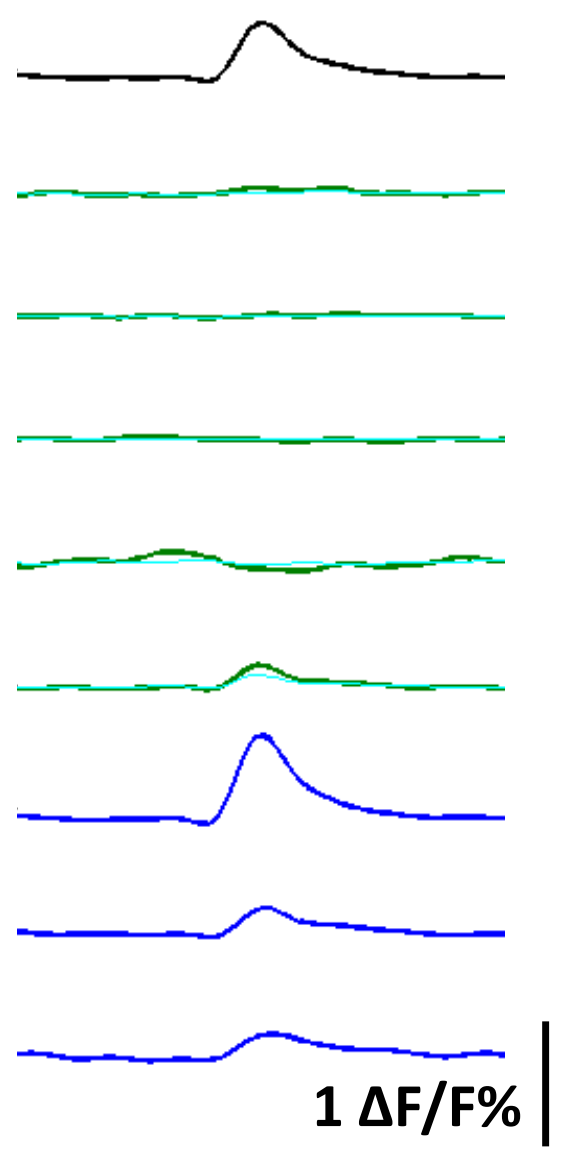
Figure 6



\section{B}

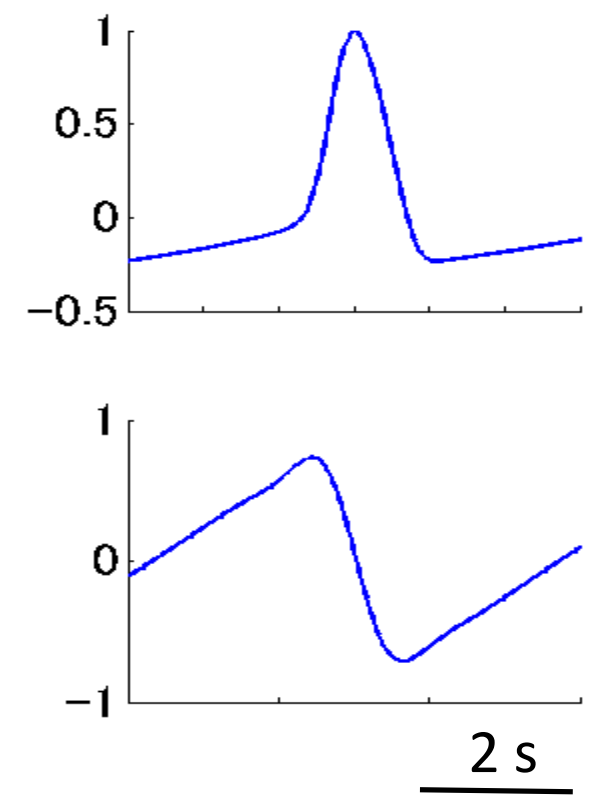

